- Review Article

\title{
Probability or Reasoning: Current Thinking and Realistic Strategies for Improved Medical Decisions
}

\author{
Yogarabindranath Swarna Nantha* \\ Primary Care Research Initiative and Methods Education Department (PRIMED), Seremban Primary Care Clinic, Seremban, Malaysia
}

\begin{abstract}
A prescriptive model approach in decision making could help achieve better diagnostic accuracy in clinical practice through methods that are less reliant on probabilistic assessments. Various prescriptive measures aimed at regulating factors that influence heuristics and clinical reasoning could support clinical decision-making process. Clinicians could avoid time-consuming decision-making methods that require probabilistic calculations. Intuitively, they could rely on heuristics to obtain an accurate diagnosis in a given clinical setting. An extensive literature review of cognitive psychology and medical decision-making theory was performed to illustrate how heuristics could be effectively utilized in daily practice. Since physicians often rely on heuristics in realistic situations, probabilistic estimation might not be a useful tool in everyday clinical practice. Improvements in the descriptive model of decision making (heuristics) may allow for greater diagnostic accuracy.
\end{abstract}

Keywords: Heuristics; Decision Making; Evidence-Based Medicine, Bias; Problem Solving 


\section{INTRODUCTION}

Decision making in the medical profession remains a science that is inseparable from the undercurrent of uncertainty. In a recent study examining diagnostic errors in medicine, a significant proportion of diagnostic errors was attributed to the cognitive processes of the doctor. ${ }^{1)}$ Advancements in cognitive sciences play a major role in decreasing human-prone medical errors. ${ }^{2)}$ Hence, there has been extensive research in the field of cognitive psychology to palliate uncertainty and reduce diagnostic errors made by clinicians.

By quantifying the elements of uncertainty, the normative Bayesian model of probability remains an important technique to increase precision in clinical decision making. ${ }^{3)}$ However, an underlying mismatch exists between the utility and practicality of this model in real-world situations. ${ }^{4)}$ Individuals incorporate little probabilistic information to account for decision making in highly naturalistic environments. ${ }^{5)}$

The purpose of this review is manifold. Primarily, this review serves as an accessible compendium of the latest theories in medical decision making for all health professionals, especially those working in the field of primary care and emergency medicine. Secondly, the article offers techniques that help improve diagnostic accuracy based on recent evidence. This article also allows health organizations and individual physicians to re-examine the possibility of implementing corrective measures in their institution based on the emerging paradigms in medical decision making and clinical reasoning. More importantly, the article draws attention to the relevance of a less probabilistic, and perhaps more practical approach by using elements of clinical reasoning, naturalistic decision making (NDM), script activation, and dual process theory when solving daily medical problems.

\section{MEDICAL DECISION MAKING UNDER UNCERTAINTY}

In general, there are three major approaches to the concept of thinking under uncertainty - the normative, descriptive, and prescriptive models. ${ }^{6}$ The normative, or rational model proposes the best possible way of achieving a particular goal or rational decision. This model is synonymous to the subjective expected utility framework, which utilizes Bayes' theorem as a standard to compare actual human decision making via mathematical equations, ${ }^{4)}$ and forms the foundation of evidence-based medical practice.

Contrary to this belief, expert physicians seldom rely on probabilistic models and often use diagnostic reasoning processes to aid in hypothesis refinement. ${ }^{7)}$ In a primary care setting, decisions must be made within a stipulated amount of time with very scarce information obtained from patients. Therefore, physicians rely primarily on heuristics (a simplification of diagnostic reasoning) because they are convenient and frequently correct although they do not reflect base rates. The greater the uncertainty in the analysis of a medical scenario, the greater the tendency of physicians to turn to diagnostic intuition rather than probabilistic models to guide their decision analysis. ${ }^{8)}$
On the other hand, the descriptive model approach describes how people normally think. ${ }^{6}$ Early proponents of this framework had reservations about the notion of making optimal choices through calculations. Contradictions to subjective utility maximization were soon discovered (for example, the Allais paradox, bounded rationality, and Prospect theory), which only serve to indicate that real-life decisions often differ from what is proposed by the principles of the normative model. ${ }^{6,9,10)}$ Physicians utilize a variant of the descriptive model, known as heuristics or rule of thumb, as a shortcut to simplify complexity of data analysis gathered during consultation. ${ }^{11)}$ According to classical decision-making theory, the efficacy of medical heuristics is often plagued by the existence of various judgment errors, called biases. ${ }^{11)}$ While the explanation and function of biases are beyond the scope of this article, discussions on biases are readily available in many books or literature reviews. ${ }^{3,11}$ Crucial to the discussion, however, is the evidence from a problem-solving perspective that challenges the conventional preconception of biases as being a faulty decision-making mechanism. ${ }^{4)}$ Results of several studies designed using problem-solving techniques suggest that clinical experts seem to use heuristics to generate accurate diagnostic hypotheses in several disciplines in medicine, despite being prone to biases. ${ }^{12-14)}$

\section{CONTEXT: CONCEPTUAL FRAMEWORK FOR A PRESCRIPTIVE MODEL}

The prescriptive model of decision making defines the rules on how one ought to think, or how a person should adopt a better decision, if not the best decision. ${ }^{6}$ The prescriptive model is a corrective design or an intervention that influences actual thinking, bringing it into closer agreement with the normative model. ${ }^{6)}$ In medicine, the prescriptive model represents (1) useful heuristics that have been widely tested through experience in a relevant field of practice, or (2) the process of being aware of the many biases that coexist in any diagnostic decision analysis.

The conceptual framework below outlines the premise of this article. A discussion of the impact of specific components of the prescriptive framework and how this framework functions in a realistic clinical environment will be dealt with in the subsequent sections of this article (Figure 1).

\section{NATURALISTIC DECISION-MAKING STRATEGIES AND THE COMPENSATORY MODEL}

Scant research effort has been mobilized towards investigating the effects of stress on medical decision making. Research coverage in this field has been limited to assessing the effects of sleep deprivation. ${ }^{15}$ Sleep deprivation seems to negatively impact physician cognition and medical performance. ${ }^{16)}$ However, these research designs were not specifically constructed to explore medical errors secondary to stress. Despite the lack of direct evidence of the effect of stress on medical decision making, researchers began designing theories (such as the 


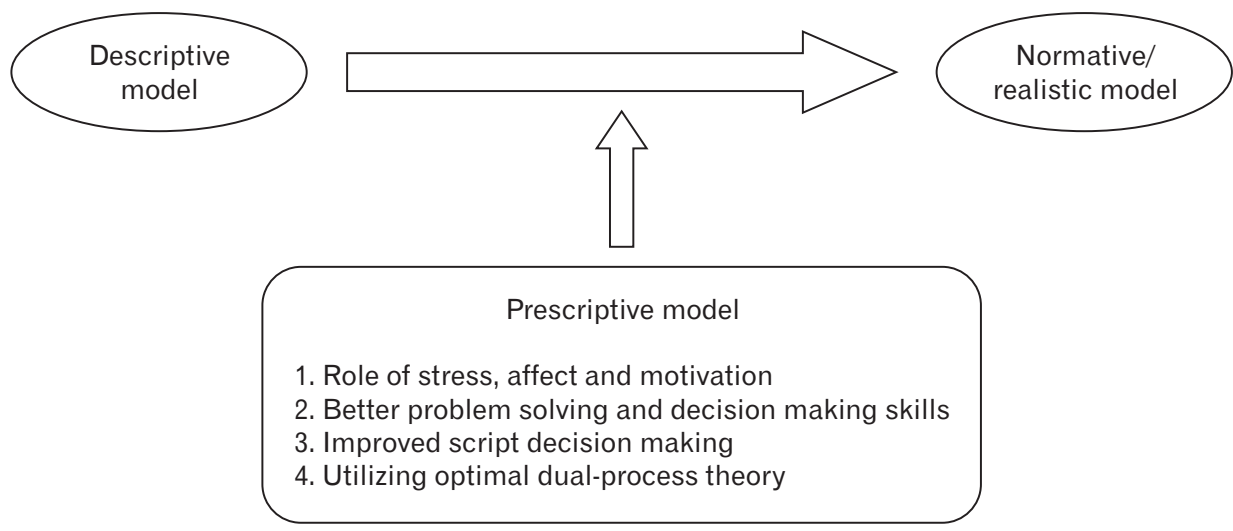

Figure 1. Conceptual framework for a prescriptive model of medical decision making.
NDM theory) that would serve to fill the gap in knowledge. ${ }^{17)}$

NDM theory revolves around the idea of gauging apparent changes in the cognition of individuals working in environments that integrate many factors, such as limited time, high stakes, vague goals, and unstable situations. ${ }^{17)}$ Studies involving NDM concepts appear to demonstrate that in stressful situations, fire commanders ${ }^{18)}$ and nurses in emergency telephone triage settings ${ }^{19)}$ rely on recognition-primed decision making., ${ }^{4,17)}$ This concept is similar to script activation, which is the automatic retrieval of a script from a vast repertoire of previous exposure to or experience with earlier cases, and is largely non analyti$\mathrm{cal}^{20,21)}$ The accuracy of these decisions is remarkably higher in experts with greater experience in the field than in novices. ${ }^{19)}$ This evidence seems to show that experts make decisions based on previous exemplars in the form of mental scripts that support decision heuristics. ${ }^{4)}$

High-stakes decision making produces high levels of perceived stress that distorts the way one thinks. ${ }^{22)}$ Under situational pressure, individuals rely more on non-compensatory methods of thinking inclined towards the use of heuristics (a category-based inferential technique). This situation arises because there is an increase in cognitive load and limited processing time. ${ }^{23,24)}$ The compensatory model (an attribute-based technique closely resembling the normative model) pursues detailed processing of individual pieces of information that could only be undertaken when there is little time constraint or less cognitive load on the individual. ${ }^{25)}$ Similarly, cues arising from emotions and changes in affect could alter decisions that have major outcomes. ${ }^{26,27)}$ These deviations from the normative model are referred to as 'affect heuristics' ${ }^{26)}$ They are a reactive response to risk without taking into account any probabilistic measure. ${ }^{22)}$ Therefore 'affect heuristics' are largely pertinent to primary care physicians who work in a very demanding environment where the absence of judicious life-saving decisions can have catastrophic outcomes for patients.

Little emphasis has been made on bridging the gap between motivational aspects of cognition and the medical decision-making process despite significant scientific contributions in both fields. In an experiment to determine the role of positive affect amongst physicians, it was found that physicians who have been induced to acquire positive affect had greater diagnostic problem-solving skills. ${ }^{28)}$ Anchoring bias in decision making is significantly less common in physicians with positive affect. ${ }^{29)}$ The cognitive evaluation theory of motivation posits that the primary components of intrinsic motivation (autonomy, competency, and relatedness) are paramount to an individual's psychological needs. ${ }^{30)}$ Hence, interventions designed to improve positive affect through intrinsic motivation could allow physicians to make better decisions in complex situations. ${ }^{31)}$

\section{STRATEGIES FOR BETTER PROBLEM-SOLVING AND DECISION-MAKING SKILLS IN CLINICAL REASONING}

\section{The Role of Hypothesis Refinement}

In clinical reasoning, two main approaches underpin the fundamentals of diagnostic thinking in medicine: problem-solving strategies and standard decision-making analysis. ${ }^{32)}$ Evaluations based on the concepts of problem-solving strategies show that expert physicians not only generate quicker hypotheses but also form diagnostic plans with greater efficacy than novices. ${ }^{11,33)}$ Also, the mechanics of problem-solving in medicine suggest that it is necessary for hypotheses formation to undergo certain crucial steps before a diagnosis is deemed valid. ${ }^{7)}$ These steps include hypotheses generation, hypotheses refinement, data verification, and finally, a working diagnosis. ${ }^{7)}$ Flaws in any of these steps could lead to a faulty provisional diagnosis.

The implications of these theories are best described in the form of a routine clinical example. It is known that experts are better than novices at identifying salient findings that might provide a more holistic view of a case. ${ }^{11)}$ In other words, experts are better at hypothesis refinement, by using memories as diagnostic criteria when assessing a particular case. This is evidenced in domain-specific disciplines, where a simple set of clinical tests and historical data may be sufficient to correctly identify a disease ${ }^{34)}$ Furthermore, conditions that cannot be easily detected by biological markers will naturally require the act of eliciting a specific set of information pertaining to clinical symptoms and disease progression to enhance hypotheses refinement. ${ }^{35}$ 


\section{Choosing Heuristics over the Standard Decision-Making Strategy}

As discussed earlier, the traditional decision-making strategy requires strict compliance with the normative model (Bayes' theorem). Contrary to this rule, most physicians still make revisions to their diagnostic opinion through heuristics. ${ }^{11)}$

Recent studies suggest that in situations of uncertainty as seen in medicine, the role of medical decision-making theory in daily practice may be negligible. ${ }^{36)}$ It is thought that, in medical decisions, mathematical calculations may not be crucial to the evidence-based model. ${ }^{37)}$ Therefore, heuristics seem to be inescapable and indispensable tool in the realm of medicine, despite their evident disadvantages.

The evidence stated above suggests that the construction of a simple, easy-to-recall heuristics could be crucial to decision making in medicine. For example, by using a fast-and-frugal tree shortcut, patients with angina have been screened with perhaps better accuracy than by applying a probabilistic model. ${ }^{38)}$ Similarly, in the evaluation of the decision to commence macrolide treatment for community-acquired pneumonia in children, the fast-and-frugal method correctly identified $72 \%$ of high-risk cases. ${ }^{39)}$ Thus, simple heuristics may have some value in making therapeutic decisions without relying on more

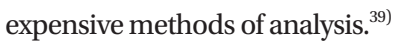

\section{IMPROVED SCRIPT DECISION MAKING}

\section{Scripts as a Non-Probabilistic Alternative in Decision Making}

Scripts seem to be a departure from the probabilistic model of evidence-based practice. Scripts describe how physicians routinely gather information from patients' complaints and recognize a familiar pattern of a particular illness. Scripts influence physicians by guiding them to formulate treatment plans without much deliberation on probabilities or utility. ${ }^{15)}$ Therefore, it is widely believed that diagnostic reasoning is highly dependent on decision scripts. ${ }^{40)}$

Decision scripts allow physicians to rely on reasoning skills to arrive at a proper clinical diagnosis, even if there are no prototypical or suitable instances available. ${ }^{40)}$ When presented with clinical vignettes, the treatment choice or perceived treatment benefit chosen by critical care physicians had little to do with prognostic probabilities. ${ }^{15,41)}$ Instead, physicians seem to rely on decision scripts to help steer them towards making a correct decision. ${ }^{15,41)}$ This action requires script activation, a phenomenon that occurs almost effortlessly in a non-analytical manner and is based on pattern recognition or instances from previous clinical experience. ${ }^{20,21)}$ One study argues that the accuracy of these decision scripts could be augmented by learning a script through exposure to analytic aids. ${ }^{15,38)}$ Through this intervention, it was found that diagnostic aids were no longer required when dealing with real-time clinical situations. ${ }^{15,38)}$

\section{Recall Advantage through Scripts}

A clinical decision can be rendered faulty if one focuses solely memo- rizing a specific script containing clinical features of a disease entity. The untenable structure of memory fails to retain such information over the course of time or with increasing case difficulty. ${ }^{42)}$ However, memory can be improved by emphasizing the mastery of knowledge in basic sciences. It was found that clinical accuracy did not diminish profoundly with time in subjects who utilized basic sciences to find coherence in a set of clinical features, in contrast to subjects who utilized epidemiological probabilities to reach the same conclusion. ${ }^{43)}$

Decision scripts are also influenced by exemplar models of categorization retrieved from past experience of discernible environmental cues. ${ }^{44)}$ However, depending on the way they are acquired, exemplars can have contrasting effects in a clinical setting. In visually oriented clinical domains, for example, prior exposure to examples of cases could enhance clinical diagnostic skills. ${ }^{45}$ Conversely, in another study using less memorable details such as electrocardiograms and historical data, diagnostic accuracy was reduced when family medicine residents chose to rely on a previous case scenario that bore a close resemblance to the clinical case at hand. ${ }^{46)}$

\section{Relevance of Scripts in Decision Making}

Results of these experiments consolidate the view that physicians and medical students might benefit from a more guided exposure to real case scenarios through structured training programs. These programs could foster better decision scripts and greater diagnostic accuracy. It would also be equally advisable to raise awareness amongst clinicians and students on how decision scripts could skew clinical reasoning if not utilized judiciously in certain circumstances. Unverified non-analytical decision scripts might lead to the problem of premature closure, a common diagnostic error. ${ }^{1)}$ Experienced clinicians were found to be more susceptible to this error since they assign more weight to their initial diagnosis compared to their less experienced counterparts. ${ }^{47)}$ Prevention of these errors could be achieved in part by paying attention to analytical reasoning and criteria of validity (coherence, adequacy, and parsimony) in the final steps of the diagnostic verification process. $^{7)}$

\section{PUTTING DUAL PROCESS THEORY INTO ACTION}

\section{Dual Process Theory and Heuristics}

Dual process theory suggests that heuristics are an inevitable aspect of the art of decision making. Contrary to being labeled as a potential precursor of biases, heuristics have been recently considered a fairly reasonable attempt at mental reasoning before analytical processing is activated. ${ }^{48)}$ It is assumed that the analytical system regulates heuristics (non-analytical judgments) by acting as a gateway that selectively endorses these intuitive decisions. ${ }^{49)}$ Heuristics make use of prior knowledge in pattern recognition and forming selective representations, which are later conveyed for analytic processing if required. ${ }^{48)}$ Thus, heuristics behave as default options that might or might not be inhibited by the analytical phase. ${ }^{48)}$ 


\section{Application of Dual Process Theory via Pattern Recognition and Hypothetico-Deductive Techniques}

In the field of medicine, pattern recognition and hypothetico-deductive techniques employed in clinical reasoning are consistent with the aspects of dual process theory. ${ }^{50)}$ Firstly, the intuitive system of dual process theory is represented by pattern recognition. ${ }^{20,51)}$ Both experts and novice physicians display greater clinical success when they engage in pattern recognition strategies to guide their diagnostic reasoning. ${ }^{52)}$ Secondly, hypothetico-deductive techniques are comparable to the analytical system ${ }^{50)}$ that spring into action when the intuition system fails to provide a convincing diagnostic hypothesis ${ }^{51)}$ In tandem with this stance, physicians often explore other avenues in decision making such as forward reasoning or exploration of biomedical research for better clues to a possible diagnosis. ${ }^{32)}$

Although mostly described in a dichotomous manner, there is growing evidence of the potential benefits that could be derived from the application of both analytical and non-analytical strategies in clinical practice ${ }^{53)}$ Several papers have alluded to the relevance of this theory in the field of general practice and emergency medicine. ${ }^{54-56)}$ Accurate diagnostic closure, a component of analytical processing, was found to be exemplified in the way general practitioners respond to complicated non-critical clinical cases in daily practice. ${ }^{55)}$ However, in instances involving urgent or high-stakes decision making, general practitioners intuitively searched for markers of severe illness using red flags derived from personal experience. ${ }^{55)}$ Hence, both the analytical and non-analytical components of the dual process system are dynamic and can be used flexibly depending on the nature of the situation at hand.

\section{Improving the Intuitive Process through Debiasing}

In order to allow effective compilation of information within the intuitive system 1 of dual process theory, the physician needs to identify accurate mental representations for fast recall. The integrity of heuristics relies on intricate cognitive processes. The mental representations, which do not contain faulty information nor excluding any critical information, would then be conveyed for further analysis under system $2 .^{48)}$

Hence, it is possible to implement a strategy to reduce biases which could, in turn, improve diagnostic accuracy. This technique is formally known as 'debiasing. 'Debiasing' allows for better judgments to be made without reference to probability estimation. ${ }^{50,57)}$ The view on the effectiveness of this approach has so far been mixed. ${ }^{58)}$ However, it has been argued that cognitive forcing strategies could be used to promote awareness in physicians and thus counter common cognitive pitfalls that could lead to biases. ${ }^{59)}$ This step could be achieved by encouraging physicians to consider alternatives to a clinical problem by constantly checking if other probable entities could account for the information gathered during a consultation. ${ }^{60)}$ Biases could also be reduced by decreasing cognitive overload on working memory by using cognitive aids such as mnemonics, algorithms, or clinical practice guidelines. ${ }^{60)}$

\section{Improving the Analytical Process through Metacognition}

The ability of system 2 to monitor the activities of system 1 needs to be kept in a strict balance. The response of cognition is influenced by various determinants and these have the potential to disrupt the performance of system $2{ }^{61,62)}$ These factors, which are numerous, include the affective state of the physician, past experience, ambient conditions, fatigue/sleep debt, and the characteristics of patients. ${ }^{62}$

Metacognition is seen as a possible strategy to attenuate this problem. Metacognition is simply defined as thinking about one's own thinking. ${ }^{63)}$ By heightening metacognition, physicians would be able to adopt a reflective approach to making clinical decisions so that biases may be reduced. ${ }^{62)}$ In a realistic and highly demanding field such as medicine, physicians could be taught to step back for a moment from a clinical scenario to examine their thoughts and affective processes. ${ }^{62)}$ Despite its promising outlook, there has been a constant debate on how metacognition could improve diagnostic efficacy. Currently, metacognition remains an avenue worth exploring but requires further validation through future clinical application and feedback. ${ }^{58)}$ In a more recent study designed to explore this premise, metacognition was seen to enhance quick and accurate reasoning skills during later consultations. $^{64)}$

\section{CONCLUSION}

In the rapidly evolving realm of medical decision making, there should be some reflection on how pre-existing research could contribute to current beliefs on diagnostic accuracy. Although this article had been critical of the role of evidence-based medicine in routine clinical practice, this should not translate into a total abandonment of this valid methodology. Instead, a pluralistic view should be adopted so that medical decisions can be maintained at an equilibrium between practicality and efficiency.

\section{CONFLICT OF INTEREST}

No potential conflict of interest relevant to this article was reported.

\section{REFERENCES}

1. Graber ML, Franklin N, Gordon R. Diagnostic error in internal medicine. Arch Intern Med 2005;165:1493-9.

2. Zhang J, Patel VL, Johnson TR. Medical error: is the solution medical or cognitive? J Am Med Inform Assoc 2002;9(6 Suppl):S75-7.

3. Sox HC, Blatt MA, Higgins MC, Marton KI. Medical decision making. 2nd ed. Philadelphia (PA): American College of Physicians; 2006.

4. Patel VL, Kaufman DR, Arocha JF. Emerging paradigms of cognition in medical decision-making. J Biomed Inform 2002;35:52-75.

5. Huber O, Wider R, Huber OW. Active information search and complete information presentation in naturalistic risky decision tasks. Acta Psychol 1997;95:15-29.

6. Baron J. Thinking and deciding. 4th ed. Cambridge: Cambridge University Press; 2007. 
7. Kassirer J, Wong J, Kopelman R. Learning clinical reasoning. Philadelphia (PA): Wolters Kluwer Health; 2010.

8. Hall KH. Reviewing intuitive decision-making and uncertainty: the implications for medical education. Med Educ 2002;36:216-24.

9. Kahneman D, Tversky A. Prospect theory: an analysis of decision under risk. Econometrica 1979;47:263-92.

10. Berk JB, Hughson E, Vandezande K. The price is right, but are the bids?: an investigation of rational decision theory. Am Econ Rev 1996;86:954-70.

11. Elstein AS, Schwartz A. Clinical problem solving and diagnostic decision making: selective review of the cognitive literature. BMJ 2002;324:729-32.

12. Joseph GM, Patel VL. Domain knowledge and hypothesis generation in diagnostic reasoning. Med Decis Making 1990;10:31-46.

13. Lesgold A, Rubinson H, Feltovich P, Glaser R, Klopfer D, Wang Y. In: Chi MT, Glaser R, Farr MJ, editors. The nature of expertise. Hillsdale (NJ): Lawrence Erlbaum Associates; 1988. p. 311-43.

14. Kushniruk AW. Complex decision making in intensive care: role of medical expertise [dissertation]. Ottawa: National Library of Canada; 1998.

15. Hardman D, Macchi L. Thinking: psychological perspectives on reasoning, judgment and decision making. Chichester: John Wiley \& Sons; 2003.

16. Philibert I. Sleep loss and performance in residents and nonphysicians: a meta-analytic examination. Sleep 2005;28:1392-402.

17. Klein G. Naturalistic decision making. Hum Factors 2008;50:456-60.

18. Klein GA, Calderwood R. Decision models: some lessons from the field. IEEE Trans Syst Man Cybern 1991;21:1018-26.

19. Leprohon J, Patel VL. Decision-making strategies for telephone triage in emergency medical services. Med Decis Making 1995;15:240-53.

20. Norman G, Young M, Brooks L. Non-analytical models of clinical reasoning: the role of experience. Med Educ 2007;41:1140-5.

21. Eva KW. What every teacher needs to know about clinical reasoning. Med Educ 2005;39:98-106.

22. Kunreuther H, Meyer R, Zeckhauser R, Slovic P, Schwartz B, Schade C, et al. High stakes decision making: normative, descriptive and prescriptive considerations. Mark Lett 2002;13:259-68.

23. Kruglanski AW, Freund T. The freezing and unfreezing of lay-inferences: effects on impressional primacy, ethnic stereotyping, and numerical anchoring. J Exp Soc Psychol 1983;19:448-68.

24. Gilbert DT, Hixon JG. The trouble of thinking: activation and application of stereotypic beliefs. J Personal Soc Psychol 1991;60:509-17.

25. Hess TM, Bolstad CA. Category-based versus attribute-based processing in different-aged adults. Aging Neuropsychol Cogn 1998;5:27-42.

26. Slovic P, Finucane M, Peters E, Macgregor DG. The affect heuristics. In: Gilovich T, Griffin DW, Kahneman D, editors. Heuristics and biases: the psychology of intuitive judgement. Cambridge: Cambridge University Press; 2002. p. 397-421.

27. Loewenstein GF, Weber EU, Hsee CK, Welch N. Risk as feelings. Psychol Bull 2001;127:267-86.

28. Estrada CA, Isen AM, Young MJ. Positive affect improves creative problem solving and influences reported source of practice satisfaction in physicians. Motiv Emot 1994;18:285-99.

29. Estrada CA, Isen AM, Young MJ. Positive affect facilitates integration of information and decreases anchoring in reasoning among physi- cians. Organ Behav Hum Decis Process 1997;72:117-35.

30. Ryan RM, Deci EL. Self-determination theory and the facilitation of intrinsic motivation, social development, and well-being. Am Psychol 2000;55:68-78.

31. Isen AM. An influence of positive affect on decision making in complex situations: theoretical issues with practical implications. J Consum Psychol 2001;11:75-85.

32. Patel VL, Arocha JF, Zhang J. Thinking and reasoning in medicine. In: Holyoak KJ, Morrison RG, editors. Cambridge handbook of thinking and reasoning. Cambridge: Cambridge University Press; 2005. p. 72750.

33. Barrows HS, Norman GR, Neufeld VR, Feightner JW. The clinical reasoning of randomly selected physicians in general medical practice. Clin Invest Med 1982;5:49-55.

34. Johnston RL, Seller MJ, Behnam JT, Burdon MA, Spalton DJ. Dominant optic atrophy: refining the clinical diagnostic criteria in light of genetic linkage studies. Ophthalmology 1999;106:123-8.

35. Litvan I, Bhatia KP, Burn DJ, Goetz CG, Lang AE, McKeith I, et al. Movement Disorders Society Scientific Issues Committee report: SIC Task Force appraisal of clinical diagnostic criteria for Parkinsonian disorders. Mov Disord 2003;18:467-86.

36. Croskerry P. The theory and practice of clinical decision-making. Can J Anesth 2005;52(Suppl 1):R1-8.

37. Loftus SF. Language in clinical reasoning: learning and using the language of collective clinical decision making [Internet]. Sydney: University of Sydney; 2006 [cited 2017 Feb 10]. Available from: http://ses.library.usyd.edu.au/handle/2123/1165.

38. Green L, Mehr DR. What alters physicians' decisions to admit to the coronary care unit? J Fam Pract 1997;45:219-26.

39. Fischer JE, Steiner F, Zucol F, Berger C, Martignon L, Bossart W, et al. Use of simple heuristics to target macrolide prescription in children with community-acquired pneumonia. Arch Pediatr Adolesc Med 2002;156:1005-8.

40. Custers EJ, Regehr G, Norman GR. Mental representations of medical diagnostic knowledge: a review. Acad Med 1996;71(10 Suppl):S55-61.

41. Elstein AS. Heuristics and biases: selected errors in clinical reasoning. Acad Med 1999;74:791-4.

42. Woods NN. Science is fundamental: the role of biomedical knowledge in clinical reasoning. Med Educ 2007;41:1173-7.

43. Woods NN, Neville AJ, Levinson AJ, Howey EH, Oczkowski WJ, Norman GR. The value of basic science in clinical diagnosis. Acad Med 2006;81(10 Suppl):S124-7.

44. Medin DL, Altom MW, Murphy TD. Given versus induced category representations: use of prototype and exemplar information in classification. J Exp Psychol Learn Mem Cogn 1984;10:333-52.

45. Regehr G, Cline J, Norman GR, Brooks L. Effect of processing strategy on diagnostic skill in dermatology. Acad Med 1994;69(10 Suppl):S34-6.

46. Hatala R, Norman GR, Brooks LR. Influence of a single example on subsequent electrocardiogram interpretation. Teach Learn Med 1999;11:110-7.

47. Eva KW, Cunnington JP. The difficulty with experience: does practice increase susceptibility to premature closure? J Contin Educ Health Prof 2006;26:192-8.

48. Evans JS. Dual-processing accounts of reasoning, judgment, and social cognition. Annu Rev Psychol 2008;59:255-78. 
49. Evans JS. The heuristic-analytic theory of reasoning: extension and evaluation. Psychon Bull Rev 2006;13:378-95.

50. Higgs J, Jones MA. Clinical decision making and multiple problem spaces. In: Higgs J, Jones MA, Loftus S, Christensen N, editors. Clinical reasoning in health professions. 3rd ed. Amsterdam: Elsevier Butterworth-Heinemann; 2008. p. 3-18.

51. Pelaccia T, Tardif J, Triby E, Charlin B. An analysis of clinical reasoning through a recent and comprehensive approach: the dual-process theory. Med Educ Online 2011;16:1-9. https://doi.org/10.3402/meo. v16i0.5890.

52. Coderre S, Mandin H, Harasym PH, Fick GH. Diagnostic reasoning strategies and diagnostic success. Med Educ 2003;37:695-703.

53. Eva KW, Hatala RM, Leblanc VR, Brooks LR. Teaching from the clinical reasoning literature: combined reasoning strategies help novice diagnosticians overcome misleading information. Med Educ 2007;41:11528.

54. Balla JI, Heneghan C, Glasziou P, Thompson M, Balla ME. A model for reflection for good clinical practice. J Eval Clin Pract 2009;15:964-9.

55. Balla J, Heneghan C, Thompson M, Balla M. Clinical decision making in a high-risk primary care environment: a qualitative study in the UK. BMJ Open 2012;2:e000414.

56. Glatter RD, Martin RE, Lex J. How emergency physicians think. Proceedings of the fourth Mediterranean Emergency Congress (MEMC
IV); 2007 Sep 15-19; Sorrento, Italy. Aartselaar: The European Society for Emergency Medicine; 2007.

57. Plous S. The psychology of judgement and decision making. Philadelphia (PA): Temple University Press; 1993.

58. Graber M. Metacognitive training to reduce diagnostic errors: ready for prime time? Acad Med 2003;78:781.

59. Croskerry P. Cognitive forcing strategies in clinical decisionmaking. Ann Emerg Med 2003;41:110-20.

60. Croskerry P. The importance of cognitive errors in diagnosis and strategies to minimize them. Acad Med 2003;78:775-80.

61. Croskerry P. Clinical cognition and diagnostic error: applications of a dual process model of reasoning. Adv Health Sci Educ Theory Pract 2009;14 Suppl 1:27-35.

62. Croskerry P. Cognitive and affective dispositions to respond. In: Croskerry P, Cosby KS, Schenkel SM, Wears RL, editors. Patient safety in emergency medicine. 2nd ed. Philadelphia (PA): Lippincott Williams \& Wilkins; 2009. p. 219-27.

63. Flavell JH. Metacognitive aspects of problem solving. In: Resnick LB, editor. The nature of intelligence. Hillsdale (NJ): Lawrence Erlbaum Associates; 1976. p. 231-5.

64. Marcum JA. An integrated model of clinical reasoning: dual-process theory of cognition and metacognition. J Eval Clin Pract 2012;18:95461. 